Original Paper http://ajol.info/index.php/ijbcs http://indexmedicus.afro.who.int

\title{
Influence de la flore ligneuse associée dans la production des parcs à Anacardium occidentale L. dans la communauté rurale de Djibanar (Casamance/Sénégal)
}

\author{
Ndiaye SEYDOU ${ }^{1}$, M. Charahabil MOHAMED ${ }^{1 *}$, Ousmane NDIAYE $^{1}$ et \\ Diatta MALAÏNY² \\ I'Département d'Agroforesterie, Université Assane Seck, BP 523 Ziguinchor, Sénégal. \\ ${ }^{2}$ Institut Sénégalais de Recherche Agricole (ISRA), Sénégal. \\ *Auteur correspondant ; E-mail : mcharahabil@univ-zig.sn ; Tel : (+221) 775320107
}

\section{RESUME}

Les plantations d'anacardier sont confrontées à d'énormes difficultés dues à la conjugaison de plusieurs facteurs. Dans la mise en place de ces plantations, seules quelques espèces ligneuses sont conservées dans ces plantations d'anacardier. Cette étude a pour objet d'établir l'influence des arbres associés aux parcs à base d'anacardier dans le Sud du Sénégal. La diversité et la structure du peuplement des arbres associés ont été étudiées à l'aide d'inventaire et de relevés de la végétation. L'influence de ces arbres sur la production des anacardes a été mise en évidence à l'aide d'une analyse en composantes principales (ACP). $\mathrm{Au}$ total, onze (11) espèces réparties dans six (6) familles sont maintenus dans les parcs étudiés pour une moyenne de 5 espèces par parc. L'ACP montre une nette corrélation entre le rendement ( $\mathrm{kg} / \mathrm{ha})$ et la diversité de la flore associée. Par contre, cette corrélation est négative entre le diamètre, la densité des individus et le rendement. Ces résultats montrent ainsi que la présence des ligneux dans les parcs à anacardier améliore dans une certaine mesure leur production. Cette production diminue lorsque ces arbres deviennent plus grands que les pieds d'anacarde et si leur densité est élevée. En somme, il serait important de conserver certaines espèces ligneuses telles que les légumineuses dans ces parcs en vue d'améliorer le rendement de l'anacardier et des cultures associées.

(C) 2016 International Formulae Group. All rights reserved.

Mots clés: Flore ligneuse, agroforesterie, Anacardier, rendement, influence.

\section{Influence of the associated woody flora in the production of Anacardium occidentale $\mathrm{L}$. at the parklands in the rural community of Djibanar (Casamance / Senegal)}

\begin{abstract}
Cashew plantations are confronted with huge difficulties for several factors. In the implementation of these plantations of cashew only few words species are preserved. The aim of this study is to establish the influence of trees associated with Anacardium occidentale's parklands in southern Senegal. The diversity and population structure of the associated trees were studied by using the inventory and vegetation surveys. The
\end{abstract}


influence of these trees on the productivity of cashew nuts was shown using a Principal Component Analysis (PCA). The eleven (11) species belonged to six (6) botanical families are held in parklands studied for an average of 5 species by parkland. CPA shows a clear correlation between yield ( $\mathrm{kg} / \mathrm{ha}$ ) and the diversity of the associated fauna. As against this negative correlation between the diameter, the entity of individuals and the performance. These results thus show that the presence of wood in cashew parklands improves to some extent their production. This production decreases when these trees became larger than the cashew feet and if their density is high. As a matter of fact, it would be important to preserve some ligneous species such as legumes in these parks to improve the yield and on the associated cultures.

(C) 2016 International Formulae Group. All rights reserved.

Keywords: Woody Plant, agroforestry, Anacardium occidentale L., yield, effect.

\section{INTRODUCTION}

Le Sénégal, avec une production moyenne annuelle estimée entre 13000 et 15 000 tonnes de noix de cajou est le quinzième pays producteur de noix de cajou au monde et le septième pays africain (USAID, 2006). Cette production de noix de cajou participe pour une large mesure de l'augmentation des revenus supplémentaires pour plus de 100.000 personnes vivant non seulement dans le monde rural, mais aussi en milieu urbain et à plusieurs niveaux : opérations de production, collecte, transport, post-récolte, transformation et exportation des produits qui sont d'une grande importance pour le pays (USAID, 2006). La plupart des estimations placent les produits de l'anacardier comme une source de revenu supplémentaire des agriculteurs dans les grandes zones de production comme le Balantacounda dans région de Sédhiou (Ndiaye, 2014). Les superficies emblavées pour les plantations d'anacardier au Sénégal varient de 40000 à 50000 ha. Ces plantations sont caractérisées par leur faible rendement $250 \mathrm{~kg} / \mathrm{ha}$ à $400 \mathrm{~kg} / \mathrm{ha}$ (Ndiaye, 2014), contrairement, en Guinée Bissau dont le rendement est certainement le plus important en Afrique avec $1200 \mathrm{~kg} / \mathrm{ha}$ (Tandjiekpon, 2005). Comme dans toutes les plantations agroforestières, les planteurs associent quelques ligneux pérennes dans ces parcs (Ndiaye, 2014). Ces espèces associées à l'anacarde sont entretenues par ces derniers lors des défrichements pour leurs vertus thérapeutiques, alimentaires, commerciales et spirituels (Wala et al., 2005). La question des interactions entre les ligneux pérennes et les cultures associées a toujours animé les débats de l'écologie fonctionnelle dans les zones sahéliennes et soudano-sahéliennes (Ndiaye, 2014). De nombreux travaux ont été menés pour comprendre la structure et le fonctionnement de ces milieux. La teneur en eau du sol (Pansu et al., 2003), les nutriments du sol (Zougmoré et al., 2004), l'influence du type de sol (Hemmati, 2009), du climat (Sagna, 2005), du feu (Marcel et al., 2001) et de la pâture ont été étudiés. De même, l'étude de leur dynamique (Diouf et al., 2002) a été entreprise. Il est important de déterminer l'influence de ces arbres dans la production des cultures associées dans ces plantations. Dans cette étude, il s'agit d'évaluer le déterminisme de la diversité et la structuration de cette flore dans la production des parcs à Anacardium occidentale $\mathrm{L}$. dans la communauté rurale de Djibanar en basse Casamance.

\section{MATERIEL ET METHODES \\ Le site d'études \\ Le cadre physique}

Situé dans la latitude $12^{\circ} 32^{\prime} 33^{\prime \prime} \mathrm{N}$ et longitude $15^{\circ} 48^{\prime} 38^{\prime \prime} \quad \mathrm{W}$ à 34 mètres d'altitude (Figure 1) dans le département de Goudomp (Région de Sédhiou), la communauté rurale de Djibanar est un territoire cosmopolite où cohabitent plusieurs ethnies dont les plus représentatifs sont les Balantes et les Mandingues. C'est également une zone à forte potentialité agricole. L'économie principale est basée sur l'agriculture, la pêche et l'élevage qui 
constituent les bases du développement économique de cette communauté rurale.

\section{Le climat}

Le climat de la région naturelle de la

Casamance est déterminé par deux éléments essentiels dont les caractéristiques confèrent à cette région son originalité. Il s'agit d'une part de sa situation en domaine climatique soudanien atlantique à pluviométrie relativement importante et d'autre part, l'alternance sur cet espace de trois flux de vents (alizé maritime, harmattan et mousson) dont le déplacement est facilité par la platitude du relief (Sagna, 2005). Les précipitations annuelles varient entre 600 $\mathrm{mm}$ et $1775 \mathrm{~mm}$. La pluviométrie est caractérisée par une grande variabilité des précipitations entre 1982 et 2012 (Figure 2) avec une pluviométrie moyenne annuelle de $1063 \mathrm{~mm}$. Il existe comme dans tous les domaines soudano-guinéens deux saisons bien marquées :

- une saison sèche qui s'étale sur environ huit (08) mois (novembre à juin) ;

- une saison des pluies ou hivernage qui dure quatre (04) mois (juillet à octobre).

Les températures sont généralement élevées. Les moyennes annuelles oscillent entre 21 ${ }^{\circ} \mathrm{C}$ et $37{ }^{\circ} \mathrm{C}$. Ces températures sont très élevées entre le mois de mars et juin.

\section{Méthodologie de l'étude}

\section{Matérialisation des plantations}

Pour décrire l'étendue des superficies des plantations d'anacardier de la zone d'étude, les images landsat 2013 ont été exploitées. Après traitement de ces images satellites avec les logiciels ENVI 4.5 puis avec ArcGIS 10, les différentes caractéristiques de la zone en termes de couverture végétale ont été décrites (Figure 3).

Sur cette carte, deux grands types de végétations ont été identifiés à savoir les plantations d'anacardier et les autres formations forestières denses. Une vérification avec Google Earth puis sur le terrain ont permis d'établir une relation existante entre les différentes teintes de l'image. La technique d'interprétation des images satellites est bien adaptée aux fins de vérification de l'occupation du sol, lorsqu'elle est associée à de l'imagerie numérique. Cette carte permet de visualiser les espaces occupés par les plantations d'anacardier dans la zone d'étude.

\section{Inventaire}

Les données de la flore associée à l'anacarde ont été collectées grâce à un inventaire forestier. Des placettes circulaires de $20 \mathrm{~m}$ de rayon ont été installées au niveau de chaque plantation. Sur les six villages de l'échantillon, Baconding (S1), Birkama (S2), Djibanar (S3), Goudomp (S4), Kaour (S5), Kounayang (S6), deux plantations de deux (2) hectares ont été choisies au hasard dans chaque site sans distinction préalable dans lesquelles quatre (4) placettes sont installées de manière aléatoire par site de deux (2) hectares. Au total, douze (12) plantations ont été évaluées soit 48 placettes installées. Les paramètres dendrométriques et de recouvrement mesurés dans ces plantations concernent la hauteur, le diamètre, largeur $\mathrm{du}$ houppier des arbres et les écartements entre les arbres.

Le diamètre des arbres est mesuré à $1,30 \mathrm{~m}(\mathrm{DBH}) \mathrm{du}$ sol à l'aide d'un compas forestier. La hauteur des arbres est mesurée à l'aide d'un dendromètre SUNTOO. La largeur moyenne du houppier et la distance entre les arbres ont été mesurées à l'aide d'un ruban gradué de $50 \mathrm{~m}$. Pour la largeur moyenne du houppier, deux mesures croisées sont prises au niveau de chaque arbre (Est-Ouest et Nord-Sud) pour en dégager une moyenne. La hauteur de l'arbre, le diamètre des tiges et la largeur moyenne $\mathrm{du}$ houppier sont fondamentalement les éléments qui permettent de mieux cerner l'espace occupé réellement par les arbres d'anacardier pour une meilleure gestion à long terme des plantations d'anacarde (Tandjiekpon, 2005).

La diversité spécifique des plantations arborées a été analysée à l'aide des indices couramment utilisés, à savoir la richesse spécifique (nombre d'espèces), 
l'indice de diversité de Shannon et l'indice d'équitabilité de Pielou. Les deux derniers indices sont basés sur la notion de la régularité. Les expressions des indices de diversité sont :

-l'indice de diversité de Shannon:

$\mathrm{H}=-\Sigma$ pilog2pi où:

$\mathrm{H}=$ indice de diversité de Shannon

pi $=$ proportion des individus dans l'échantillon total qui appartiennent à l'espèce $\mathrm{i}$.

Il exprime l'importance relative du nombre d'espèces abondantes dans un milieu donné. L'indice est minimum quand tous les individus appartiennent à la même espèce. Il est maximal quand chaque individu représente une espèce distincte. Exprimées en bit, les valeurs extrêmes sont comprises entre 0 (diversité très faible) et 4,5 bits environ, ou exceptionnellement plus dans le cas des échantillons de grande taille dans les communautés complexes.

La valeur de l'indice varie en fonction du nombre d'espèces présentes et de leurs proportions relatives. Ce qui rend plus exhaustif son usage pour la comparaison de deux milieux à richesse spécifique très différente. Ainsi, l'indice de régularité ou d'équitabilité (E) apparaît plus intéressant pour comparer différents milieux. -l'indice d'équitabilité de Pielou (E) : $\mathrm{E}=\frac{\boldsymbol{H}}{\log _{2} S}$ où :

$\mathrm{S}=$ nombre total des espèces dans le parc ;

$\mathrm{H}=$ indice de diversité de Shannon.

Elle varie entre 0 et 1 . L'équitabilité de Pielou (E) tend vers 0 lorsqu'il y a un phénomène de dominance. Elle tend vers 1 lorsque la répartition des individus entre les espèces est régulière.

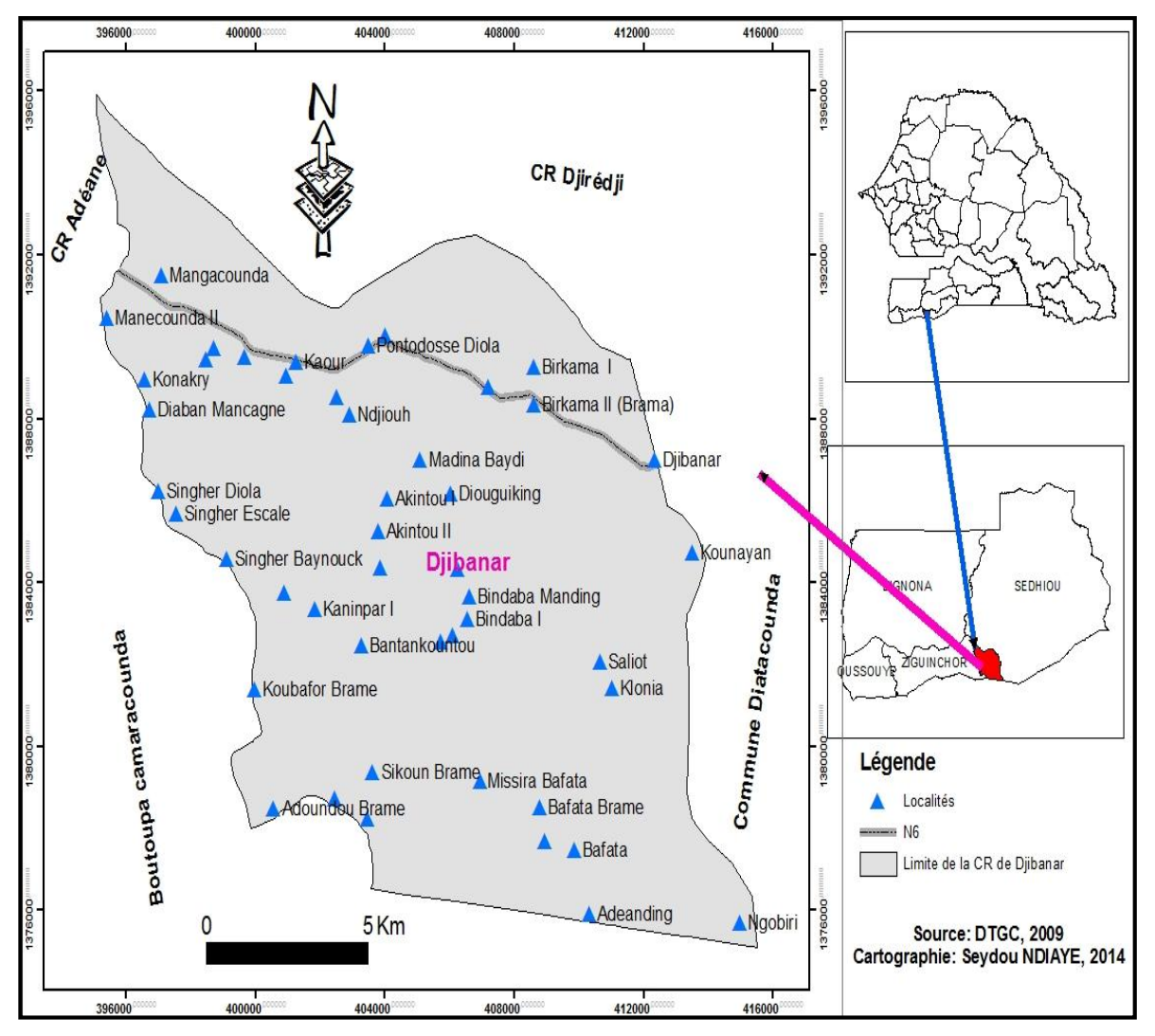

Figure 1 : Carte de localisation de la communauté rurale de Djibanar. 


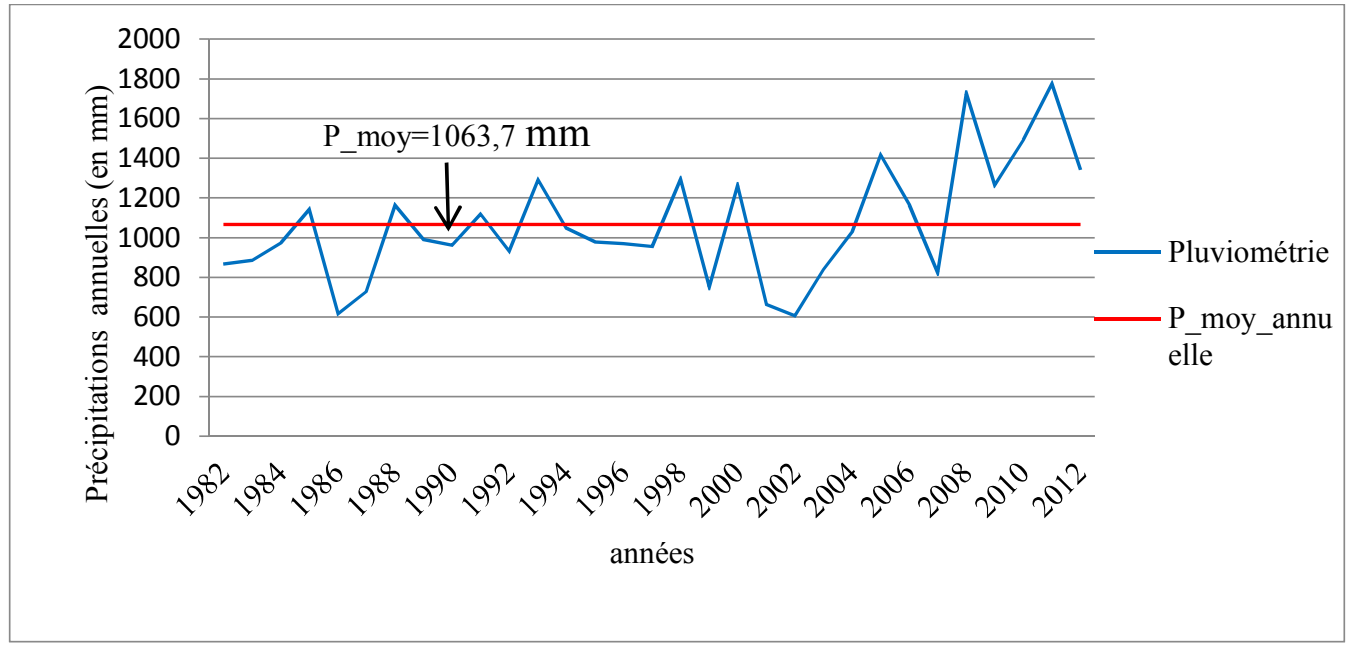

Figure 2 : Variation de la pluviométrie annuelle de la région de Sédhiou de 1982 à 2012.

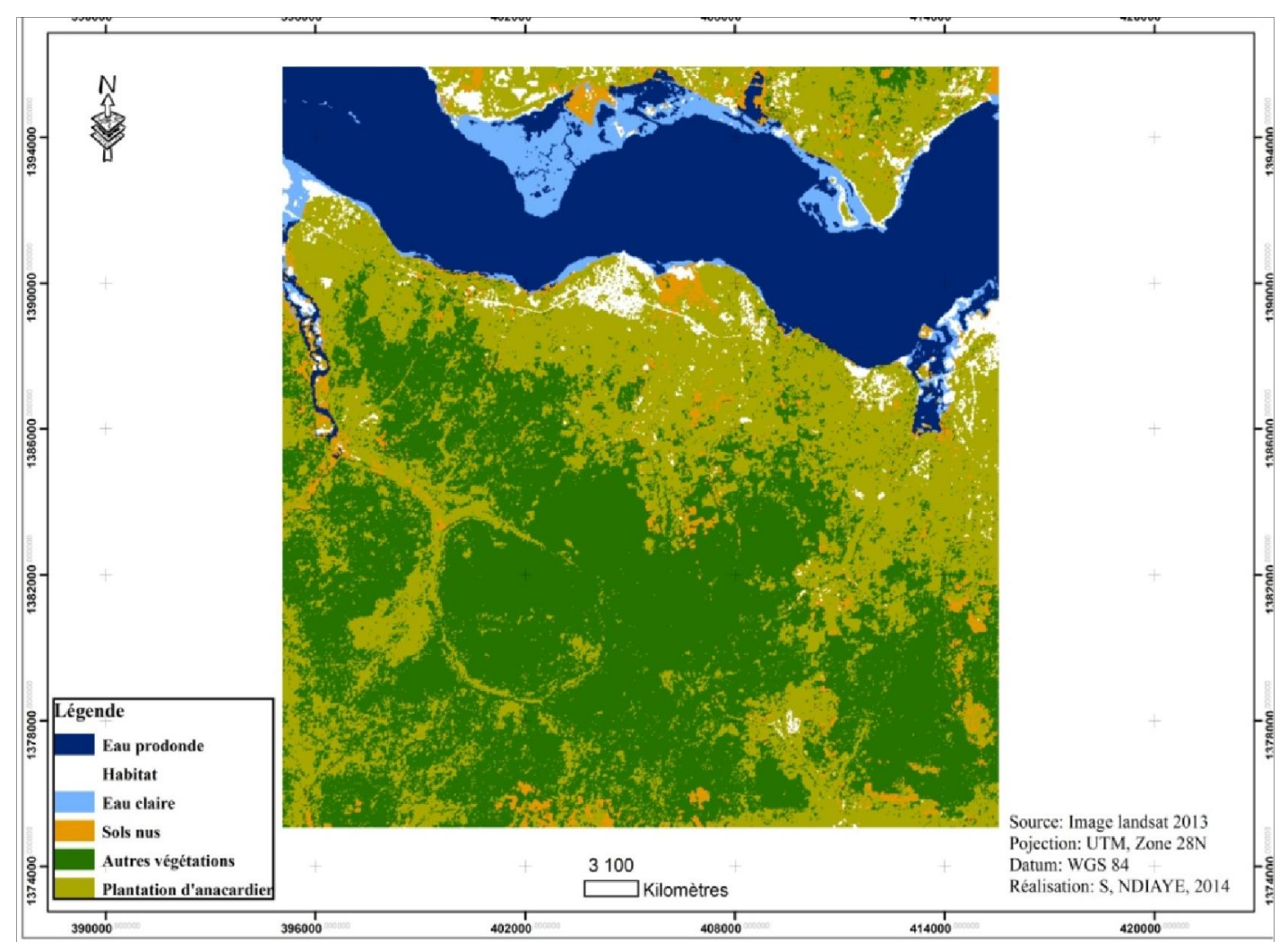

Figure 3 : Carte de matérialisation des plantations d'anacardier dans la Communauté Rurale de Djibanar. 
RESULTATS

Flore ligneuse associée

Diversité spécifique et niveau d'organisation

La flore ligneuse associée au parc à Anacardium occidentale L est constituée de onze (11) espèces ligneuses réparties dans six (6) familles (Tableau 1).

Les Fabacées sont représentées par 2 espèces, les Arécacées (2 espèces), les Méliacées (2 espèces), Malvacées (3 espèces), Anacardiacées (1 espèce) et Combrétacées ( 1 espèce). Le total des colonnes indique en plus de la diversité en espèces et en familles, le nombre d'espèces présentes dans un site et le total des lignes le nombre de site où une espèce est rencontrée. Parkia biglobosa est la seule espèce présente dans tous les sites. Quatre espèces sont cantonnées dans un seul site: il s'agit de Khaya senegalensis (S6), Guiera senegalensis (S2), Afzelia africana (S3), et Bombax costatum (S4). Le site $\mathrm{n}^{\circ} 3$ est le plus diversifié (6 espèces). Le site le plus pauvre correspond au site numéro 5 (3 espèces). La Figue 4 représente les valeurs de l'indice de diversité de Shannon et d'équitabilité de Pielou de chaque site.

Les sites S1, S2 et S3 sont caractérisés par les indices de diversité les plus élevés que les sites S4, S5 et S6. La faible diversité est exprimée dans le site S4.

\section{Importance spécifique}

Les proportions des espèces ligneuses associées aux plantations dans chaque site sont consignées dans la Figure 5.

Sur l'ensemble des sites, onze (11) espèces ligneuses sont associées au parc à Anacardium occidentale L. L'importance spécifique des ligneux associés varie d'un site à un autre. Parkia biglobosa est la plus importante pour l'ensemble des sites $(7,4 \%)$. Cette espèce est présente dans tous les sites : $(\mathrm{S} 1=20,40 \%) ; \quad(\mathrm{S} 2=24,37 \%) ; \quad(\mathrm{S} 3=$ $14,9 \%)$; $(\mathrm{S} 4=43,55 \%)$; $(\mathrm{S} 5=63,25 \%)$ et $(\mathrm{S} 6=38,05 \%)$. Dans les trois premiers sites dont en moyenne 5 espèces sont associées à l'anacarde, Borassus akeassii est la plus dominante respectivement $37 \%$ dans le site $1 ; 35,25 \%$ dans le site 2 et $32,69 \%$ dans le site 3. Dans les trois derniers sites, Parkia biglobosa est la mieux répandue avec respectivement $43,55 \%$ dans le site 4 ;
$63,25 \%$ dans le site 5 et $38,05 \%$ dans le site 6. Les espèces les plus faiblement représentées par site sont: Bombax costatum, Guiera senegalensis et Khaya senegalensis.

Influence de la flore ligneuse sur la production

Les données du Tableau 2 résument les différentes variables dans chaque site. La première colonne correspond au rendement $(\mathrm{kg} / \mathrm{ha})$ dans les différents sites et les autres colonnes, les variables relatives à la flore.

Une matrice de corrélation basée sur le coefficient de corrélation de Pearson a été réalisée et a permis d'établir le lien entre ces différentes variables (Tableau 3).

Les corrélations positives les plus élevées sont exprimées entre la richesse spécifique moyenne et l'indice de Shannon (0.751), ce qui est tout à fait normal et qui indique que ces deux paramètres évoluent ici dans le même sens. Ensuite, entre la production et la diversité $\mathrm{S}(0.670)$ et RS (0.511). La diversité des arbres associés au parc à anacarde aurait donc une à une influence positive sur le rendement. Les corrélations négatives s'expriment respectivement entre la production et le diamètre des arbres associées $(-0,543)$ et la production et la densité de ces mêmes arbres $(-0,304)$. Cela indique que plus les arbres sont âgés avec une densité importante et plus ils influencent négativement sur le rendement de l'anacarde.

L'analyse en composantes principales (Figure 6) montre que par rapport à l'axe F1, les variables "densité (De)", "la richesse spécifique (RS)", "l'indice de Shannon (S)" sont avec le rendement $(\mathrm{P})$ " situés en abscisse positive et s'opposent au variable diamètre situé en abscisses négatives. Par rapport aux sites, il y a une opposition entre $\mathrm{S} 1, \mathrm{~S} 2$ et $\mathrm{S} 3$ en abscisses positives et $\mathrm{S} 4, \mathrm{~S} 5$ et $\mathrm{S} 6$ en abscisses négatives. Cet axe permet de confirmer l'opposition entre diamètres des arbres associés et rendement.

L'axe F2 apporte une information par rapport à la particularité des sites. Ainsi, le site S3 est caractérisé par les plus forts rendements, les sites S1 et S2 par les densités élevées des arbres associés et les sites S4 et S6 par les plus gros diamètres. 
Tableau 1 : Diversité spécifique et niveau d'organisation par site.

\begin{tabular}{|c|c|c|c|c|c|c|c|c|}
\hline \multirow[b]{2}{*}{ Familles } & \multirow[b]{2}{*}{ Espèces } & \multicolumn{6}{|c|}{ Sites } & \multirow[b]{2}{*}{$\begin{array}{c}\text { Nombre total } \\
\text { par famille }\end{array}$} \\
\hline & & S1 & $\mathbf{S 2}$ & S3 & S4 & S5 & S6 & \\
\hline Anacardiacées & Mangifera indica & 1 & 1 & 1 & 0 & 0 & 0 & 3 \\
\hline \multirow[b]{2}{*}{ Arécacées } & Elaeis guineensis & 1 & 0 & 1 & 1 & 0 & 0 & \multirow{2}{*}{6} \\
\hline & Borassus akeassii & 1 & 1 & 1 & 0 & 0 & 0 & \\
\hline Combrétacées & Guiera senegalensis & 0 & 1 & 0 & 0 & 0 & 0 & 1 \\
\hline \multirow[b]{2}{*}{ Fabacées } & Afzelia africana & 0 & 0 & 1 & 0 & 0 & 0 & \multirow{2}{*}{7} \\
\hline & Parkia biglobosa & 1 & 1 & 1 & 1 & 1 & 1 & \\
\hline \multirow[b]{3}{*}{ Malvacées } & Ceiba pentandra & 0 & 0 & 0 & 1 & 0 & 1 & \multirow{3}{*}{7} \\
\hline & Andansonia digitata & 0 & 1 & 1 & 0 & 1 & 1 & \\
\hline & Bombax costatum & 0 & 0 & 0 & 1 & 0 & 0 & \\
\hline \multirow[b]{2}{*}{ Méliacées } & Azadirachta indica & 1 & 0 & 0 & 0 & 1 & 1 & \multirow{2}{*}{4} \\
\hline & Khaya senegalensis & 0 & 0 & 0 & 0 & 0 & 1 & \\
\hline 6 & 11 & 5 & 5 & 6 & 4 & 3 & 5 & \\
\hline
\end{tabular}

Tableau 2 : Synthèse des variables quantitatives dans chaque site.

\begin{tabular}{lccccc}
\hline Sites & P (kg/ha) & Dm $\mathbf{( c m )}$ & De (nbr/ha) & S & R S \\
\hline S1 & 440 & 24 & 230 & 1,11 & 6 \\
S2 & 455 & 9 & 236 & 0,96 & 6 \\
S3 & 800 & 12,5 & 192 & 1,23 & 7 \\
S4 & 390 & 23 & 224 & 0,46 & 5 \\
S5 & 500 & 16 & 162 & 0,7 & 4 \\
S6 & 400 & 32 & 185 & 0,75 & 6 \\
\hline$P=$ production, Dm $=$ Diamètre moven, De = Densité, $S=$ indice de Shannon, RS = Richesse spécifique moyenne.
\end{tabular}

Tableau 3 : Matrice de corrélation de Pearson (ACP normée, variances en 1/n).

\begin{tabular}{cccccc}
\hline & $\mathbf{P}(\mathbf{k g} / \mathbf{h a})$ & Dm $\mathbf{( c m})$ & De (nbr/ha) & $\mathbf{S}$ & $\mathbf{R ~ S}$ \\
\hline $\mathrm{P}(\mathrm{kg} / \mathrm{ha})$ & 1 & $-0,543$ & $-0,304$ & $\mathbf{0 , 6 7 0}$ & 0,511 \\
$\mathrm{Dm}(\mathrm{cm})$ & $\mathbf{- 0 , 5 4 3}$ & 1 & $-0,124$ & $-0,390$ & $-0,084$ \\
$\mathrm{De}(\mathrm{nbr} / \mathrm{ha})$ & $\mathbf{- 0 , 3 0 4}$ & $-0,124$ & 1 & 0,143 & 0,352 \\
$\mathrm{~S}$ & 0,670 & $-0,390$ & 0,143 & 1 & 0,751 \\
$\mathrm{R} \mathrm{S}$ & 0,511 & $-0,084$ & 0,352 & 0,751 & 1 \\
\hline
\end{tabular}




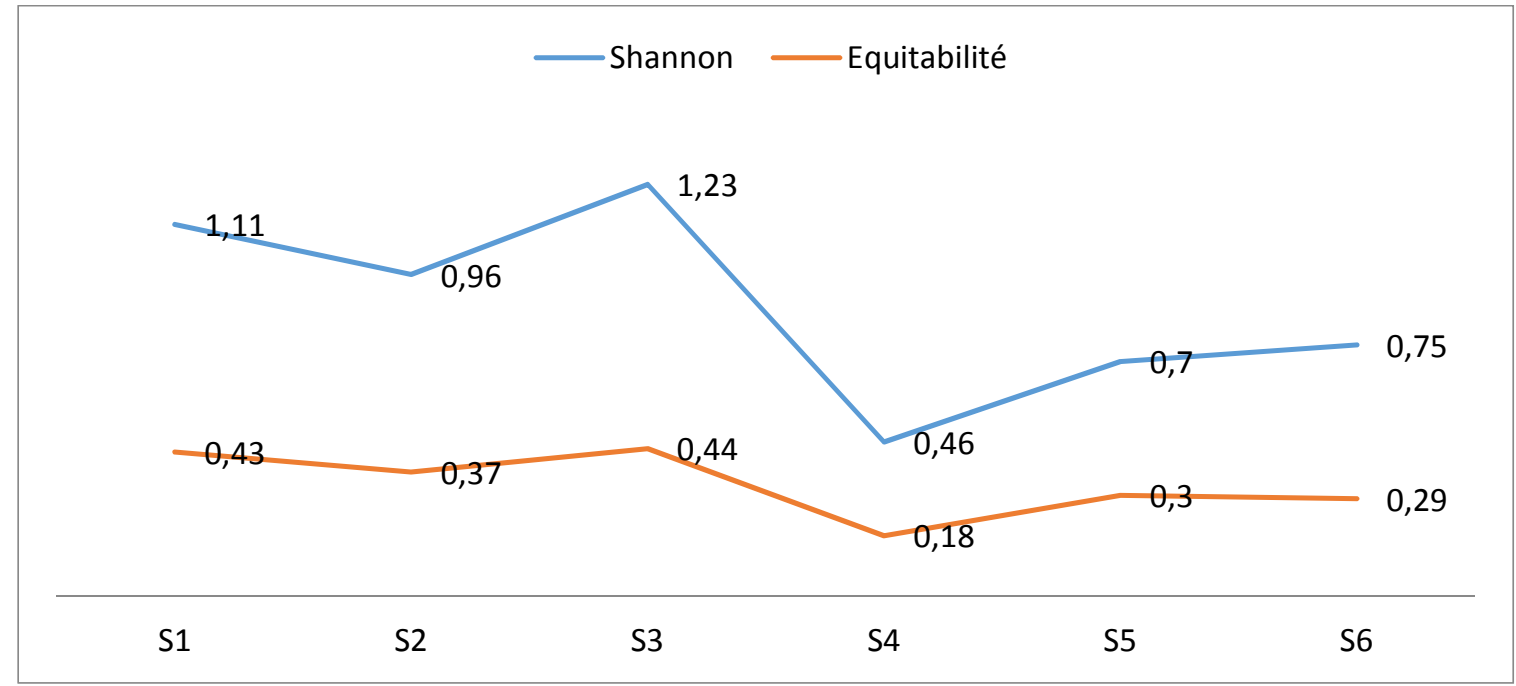

Figure 4 : Indice de diversité de Shannon et d'équitabilité de Piélou au niveau des sites.

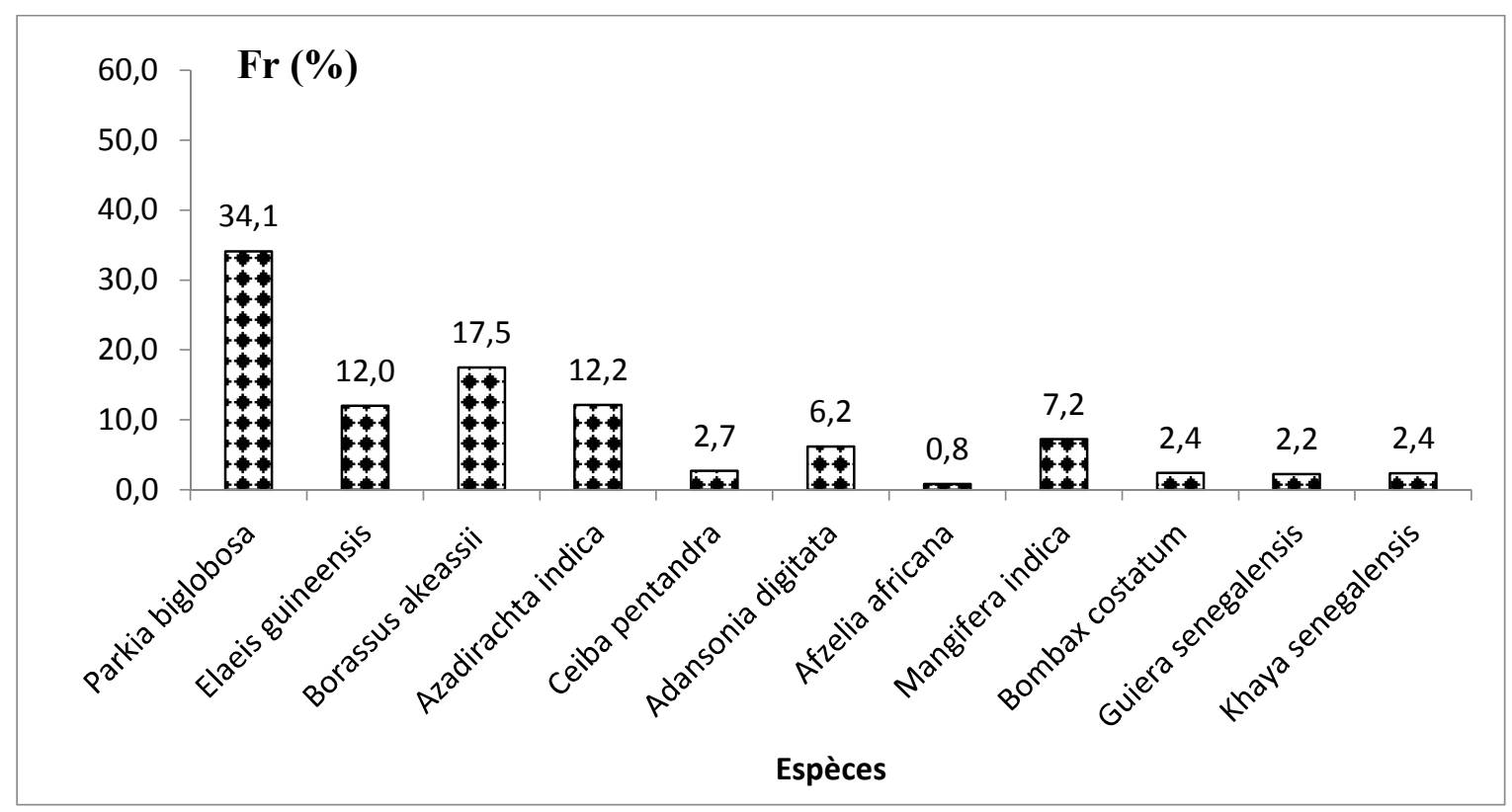

Figure 5 : Fréquence relative (\%) des ligneux associés à l'anacarde dans les parcs. 


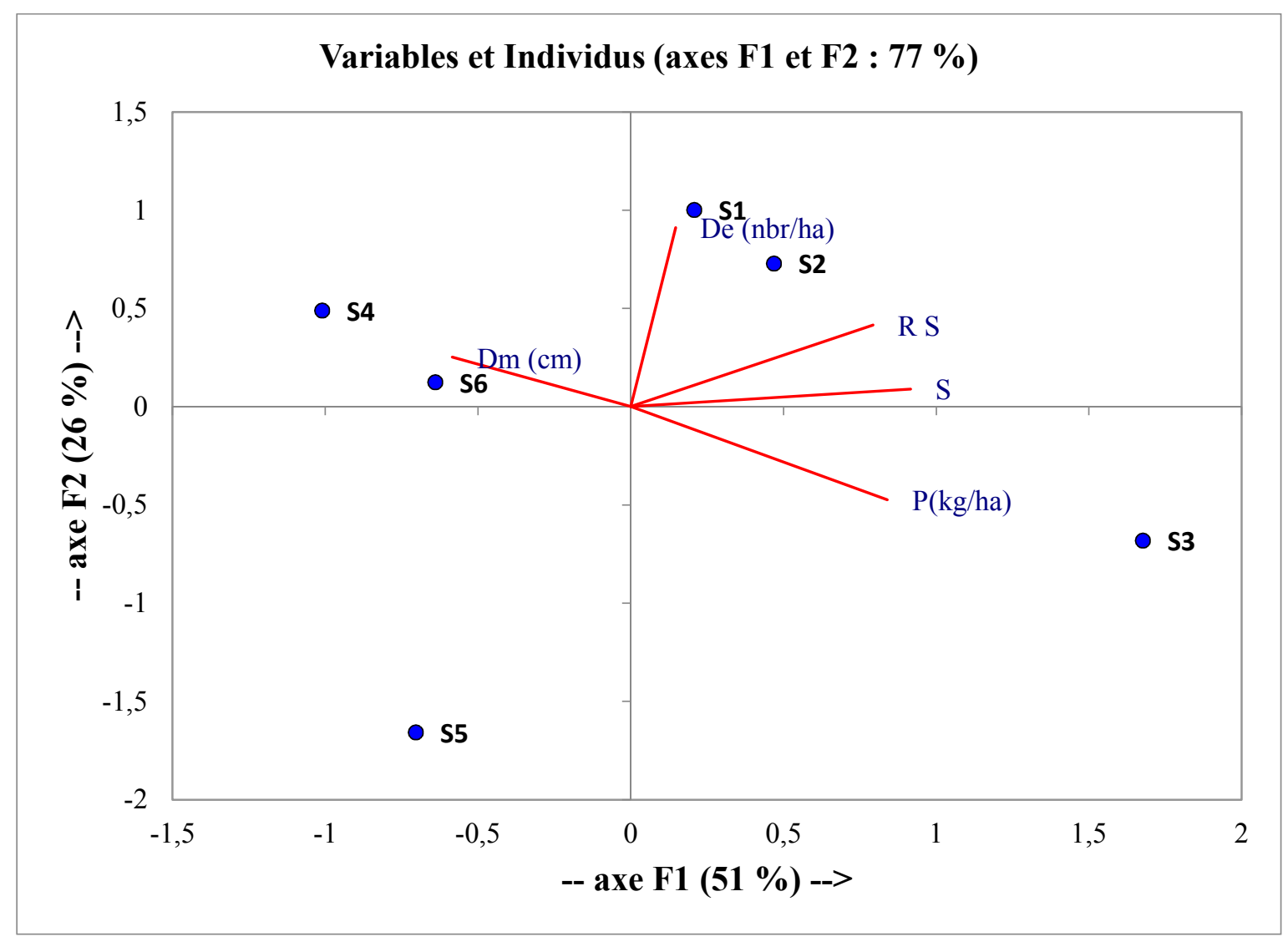

Figure 6 : Analyse en Composante Principale (ACP) de la matrice relative au Tableau 2.

\section{DISCUSSION}

\section{Diversité spécifique et Indice de diversité}

Cette étude a montré qu'au total, onze (11) espèces ligneuses sont associées aux parcs à Anacardium occidentale. En moyenne, cinq (5) espèces ligneuses sont rencontrées dans les plantations par site (Tableau 1). Des intérêts particuliers sont portés sur certaines espèces telles que le Parkia biglobosa qui présente dans tous les sites. Ceci est dû par le fait que cette espèce est traditionnellement protégée par les producteurs pour son usage agricole, pastoral et vétérinaire: les feuilles mangées par le bétail, les fleurs favorables à l'apiculture (miel brun foncé) et les fruits utilisés pour le traitement de certaines maladies telles que les diarrhées chez les volailles (vraisemblablement coccidiose) (Ndiaye, 2014).

A l'inverse, certaines espèces ne sont rencontrées que dans un seul site. Cela s'explique par le fait que seules quelques espèces utiles sont préservées lors des défrichements et entretenues dans les plantations, ce qui réduit la diversité par rapport au peuplement naturel. Ces espèces sont conservées pour leurs vertus thérapeutiques, alimentaires et commerciales (Wala et al., 2005). Le nombre d'espèces conservées dans ces plantations est inférieur aux résultats de Rouxel, (2002). Par contre, 
Soto-Pinto et al. (2000) ont émis une précision par rapport à ces faibles valeurs de diversité. Selon ces auteurs, si les pratiques à l'intérieur du système agroforestier s'intensifient ou si l'agriculteur ne permet que la croissance de quelques espèces en fonction de leur valeur économique, la biodiversité diminue. C'est pourquoi la rotation des cultures peut être nécessaire afin de permettre la régénération de certaines espèces (Okullo et Waithum, 2007).

Il existe donc une relation étroite entre la valorisation économique des plantations d'anacardier et la conservation de la diversité biologique. Ainsi, un aménagement est cependant nécessaire par le biais d'une sylviculture appropriée favorisant l'utilisation des dimensions optimales lors des semis afin de favoriser la régénération naturelle dans ces agro-forêts. On conclut alors que l'agroforesterie est plus durable en termes de conservation de la biodiversité que l'agriculture moderne. C'est du reste l'avis de (Yabi et al., 2013).

Les valeurs des différents indices de diversité varient d'une plantation à une autre. L'indice de Shannon permet la comparaison de deux milieux en terme de diversité floristique (Ngom, 2013). L'indice d'équitabilité de Pielou a la plus grande valeur dans les sites S1, S2 et S3. La valeur élevée de cet indice dans ces parcs témoigne d'un nombre élevé d'espèces conservées dans ces parcs par rapport aux trois derniers. Cela s'explique par le fait que seules quelques espèces utiles sont préservées lors des défrichements et entretenues dans les plantations, ce qui réduit la diversité par rapport au peuplement naturel (Yabi et al., 2013).
Influence des variables de diversité sur le rendement

Les résultats de l'étude montent que les sites qui sont fortement corrélés aux densités élevées ( $\geq 230$ individus/ha) sont caractérisés par un faible rendement par rapport aux sites de faible densité. L'accroissement en diamètre de l'anacardier contribue à l'augmentation de la densité au sein des plantations. C'est dire que l'accroissement du diamètre de l'anacardier rend plus dense ces plantations d'anacardier, puis que celui-ci est fortement corrélé à celui $\mathrm{du}$ houppier. Ainsi, plus le diamètre croît, plus le rendement diminue. Ces résultats confirment ceux obtenus par Tandjiekpon, (2005) ; Ndiaye (2014). La flore associée à l'anacarde dans ces parcs a un effet sur le rendement. Cette augmentation du rendement pourrait s'expliquer par le fait que ces espèces associées permettent au moment de la floraison d'attirer les pollinisateurs (abeilles, oiseaux, insectes, etc.). Cette situation profite à l'anacardier de faire une bonne pollinisation, ce qui permet d'avoir de bon rendement. Ces espèces associées à l'anacardier constituent une valeur ajoutée pour les ces planteurs, puisque leurs fruits sont commercialisés par ces derniers (Wala et al., 2005). Cependant, les cultures associées contribuent à l'amélioration des rendements au sein de ces plantations. Les légumineuses associées aux jeunes plants d'anacardier favorisent également la croissance de ces derniers (Ndiaye, 2014). Les mêmes raisons sont évoquées par Abeysinghe (2009) pour expliquer l'intérêt de l'association des cultures annuelles à l'anacardier. Les études réalisées par Opoku-Ameyaw et al. (2011) relatives à l'effet de l'association des 
cultures annuelles sur la croissance et la production de l'anacardier au Ghana ont montré que l'intervention de maïs et de sorgho ou d'arachide ont amélioré significativement la hauteur des arbres et la circonférence du tronc des anacardiers. C'est dans ce sens que l'agroforesterie doit être valorisée pour l'intensification des rendements dans ces plantations d'anacardier mais également pour la valorisation et la conservation de la diversité biologique dans ces plantations d'anacardier.

\section{Conclusion}

Cette étude a montré qu'au total, 11 espèces ligneuses appartenant à 6 familles sont conservées dans ces parcs. Dans la zone étudiée, Parkia biglobosa semble être l'espèce la plus conservée. Contrairement à ce qu'on pourrait penser, la diversité des espèces ligneuses maintenues dans les parcs à anacarde améliore leur rendement. Par contre, ces arbres ne devraient pas avoir ni des gros diamètres ni être abondants. Ces résultats montrent que l'agroforesterie est une alternative pour l'amélioration des rendements au sein de ces agro-forêts mais aussi un outil puissant pour la conservation de la diversité biologique.

\section{REFERENCES}

Abeysinghe DC. 2009. Effect of intercropping of young cashew (Anacardium occidentale L.) on land productivity. In: Recent Develop-ments in Cashew Research. Attanayaka, D.P.S.T.G., and Jayasekera, S.J.B.A. (Eds.). Proceedings of the Cashew Research Workshop held on 20 November 2009 at the Faculty of Agriculture and Plantation
Management of the Wayamba University of Sri Lanka, p.23.

Bisseleua D, Herve B, Stefan V. 2008. Plant biodiversity and vegetation structure in traditional cocoa forest gardens in southern Cameroon un-der different management. Biodiversity Conserv, 17: 1821-1835. http://link.springer. com/article/10.1007/s10531-007-92761

Daniele C, Michael K. 2011. Conservation Value of Cacao Agroforestry Systems for Terrestrial Herbaceous Species in Central Sulawesi. Indonesia. Biotropica, 43(6): 755-762. http://onlinelibrary.wiley.com/doi/10.1 111/j.1744-7429.2010.00741.x/full

Diouf M, Akpo LE, Rocheteau. 2002. Dynamique du peuplement ligneux d'une végétation sahélienne au NordSénégal (Afrique de l'ouest). Journal des Sciences, 2: 1-9.

Hemmati S. 2009. Etude de l'interaction SolVégétation-Atmosphère avec une approche couplée Thermo-HydroMécanique. Mémoire de DESS, École nationale des ponts et chaussées (France), p. 89.

Marcel H, Brice S, et Jean L. 2001. Impact des feux de brousse sur la dynamique des communautés végétales dans la forêt de Bassila (Bénin). Acta Botanica Gallica, 148: 3. http://www.tandfonline.com/doi/ abs/10.1080/12538078.2001.10515891

Ndiaye S. 2014. Caractérisation des plantations à base de Anacardium occidentale $\mathrm{L}$ dans la communauté rurale de Djibanar. Mémoire de Master, Université Assane Seck de Ziguinchor (Sénégal), p. 58. 
Ngom D. 2013. «Diversité végétale et quantification des services écosystémiques de la réserve de biosphère du Ferlo (Nord-Sénégal) », Thèse de doctorat, Université Cheikh Anta Diop de Dakar, p.172.

Okullo JBL, Waithum G. 2007. Diversity and conservation of on-farm woody plants by field types in Paromo Subcounty, Nebbi District, northwestern Uganda. Af. J. Ecol., 45: 5966. http://onlinelibrary.wiley.com/ doi/10.1111/j.1365-2028.2007.00859. $\mathrm{x} /$ full

Opoku-Ameyaw K, Oppong FK, Amoah F. M, Osei-Akoto S, Watson E. 2011. Growth and early yield of cashew intercropped with food crops in northern Ghana. J. Tropical Agri., 49(1-2): 53 - 57. www.jtropag.in/

Pansu M, Gautheyrou J, Aventurier A, Feller C, Bottner P. 2003. L'Analyse du Sol : Minéralogique, Organique et Minérale. (ed). Springer: Paris; 1012. http://www.documentation.ird.fr/hor/fdi: 010030120

Zougmoré R, Ouattara K, Mando A, Badiori O. 2004. Rôle des nutriments dans le succès des techniques de conservation des eaux et des sols (cordons pierreux, bandes enherbées, zaï et demi-lunes) au Burkina Faso. Science et changements planétaires. Sécheresse, 15(1): 41-48. http://www.jle.com/fr/revues/sec/e-docs

Rouxel C. 2002. De la biodiversité arborée au sein de terroirs de la zone semi-aride ouest-africaine : cas des parcs agroforestiers du village de M'Pébougou Sokala (région de Ségou, Mali). Mémoire DESS : Gestion des systèmes agro-sylvo-pastoraux en zones tropicales, Créteil Université de Paris-

Val-de-Marne, Créteil, p.129. http://agritrop.cirad.fr/517059/

Sagna P. 2005. «Dynamique du climat et son évolution récente dans la partie ouest de l'Afrique Occidentale ». Thèse de Doctorat d'Etat, Université Cheikh Anta Diop de Dakar, 2006. Tome I, p. 270; Tome II, p. 516.

Soto-Pinto L, Perfecto I, Castillo-H J, Caballero-N J. 2000. Shade effect on coffee production at the northern Tzeltal zone of the state of Chiapas, Mexico. Ag. Ecosystem. \& Env., 80: 91-69. http://www.sciencedirect.com/ science/article/pii/S0167880900001341

Tandjiekpon MA. 2005. Caractérisation du système agroforestier à base de l'anacardier (Anacardium occidentale Linnaeus) en zone de savane au Bénin, Mémoire de DEA, p.122.

USAID. 2006. Etude de la Filière de l'Anacardier dans la Vallée du Yamé. USAID : Mali ; 45.

Wala K, Sinsin B, Guelly KA, Kokou K, Akpagana K. 2005. Typologie et structure des parcs agro-forestiers dans la préfecture de Doufelgou (Togo). Sécheresse, 16(3) : 209-216. http://www.jle.com/fr/revues/sec/edocs/

Yabi I, Yabi F, Dadegnon S, 2013. Diversité des espèces végétales au sein des agroforêts à base d'anacardier dans la commune de Savalou au Benin. Int. J. Biol. Chem. Sci., 7(2): 696-706. http://www.ajol.info/index.php/ijbcs/art icle/view/92437. 